\title{
MS Annika: A new Cross-Linking Search Engine Supplemental Information
}

\author{
Georg J. Pirklbauer ${ }^{a}$, Christian E. Stieger ${ }^{b, c}$, Manuel Matzinger ${ }^{b}$, Stephan Winkler ${ }^{a}$, Karl Mechtler ${ }^{b}$, , \\ ${ }^{\mathrm{e}}$ and Viktoria Dorfer ${ }^{\mathrm{a}^{*}}$ \\ a University of Applied Sciences Upper Austria, Bioinformatics Research Group, Softwarepark 11, 4232 \\ Hagenberg, Austria \\ b Institute of Molecular Pathology (IMP), Vienna BioCenter (VBC), Campus-Vienna-Biocenter 1, 1030 \\ Vienna, Austria \\ c Chemical Biology Department Leibniz-Forschungsinstitut für Molekulare Pharmakologie (FMP), \\ Robert-Rössle-Strasse 10, 13125 Berlin, Germany \\ ${ }^{\mathrm{d}}$ Institute of Molecular Biotechnology (IMBA), Austrian Academy of Sciences, Vienna BioCenter (VBC), \\ Dr. Bohr-Gasse 3, 1030 Vienna, Austria
}

e Gregor Mendel Institute (GMI), Austrian Academy of Sciences, Vienna BioCenter (VBC), Dr. BohrGasse 3, 1030 Vienna, Austria

* Corresponding Author: Viktoria Dorfer, viktoria.dorfer@fh-hagenberg.at, 00435080422740 


\section{Table of Contents}

Supplementary Figure 1: The MS Annika Workflow in Proteome Discoverer

Supplementary Figure 2: Evidence Mode of MS Annika

Supplementary Figure 3: Indication Mode of MS Annika

Supplementary Table 1: Numbers of identified cross-links and CSMs for different data sets presented in this work

Supplementary Table 2: Settings for different tools mentioned in the main text

Supplementary Table 3: CSM level FDRs for MS Annika

Supplementary Figure 4: Overlap of cross-linkers for DSSO linked peptides

Supplementary Figure 5: Overlap of cross-linkers for DSBU linked peptide

Supplementary Figure 6: Runtime and memory analysis

Supplementary Figure 7: Protein interaction network created within xiView

Supplementary Figure 8: Modified Workflow for timsTOF data, using the Bruker Ion Mobility Reader

Supplementary Figure 9: Example CSM of the DSSO data set by Beveridge et al. [20], as depicted in Thermo Proteome Discoverer 2.5.

Supplementary Figure 10: Example CSM of the DSSO data set by Beveridge et al. [20], as depicted in Thermo Proteome Discoverer 2.5.

Supplementary Material 1: Pseudo-code of the main functionality of MS Annika

(MSAnnika_pseudo_code.pdf)

Supplementary Material 2: In-house R script used to create Figure 2

(MSAnnika_plots_bar_and_venn.R) 


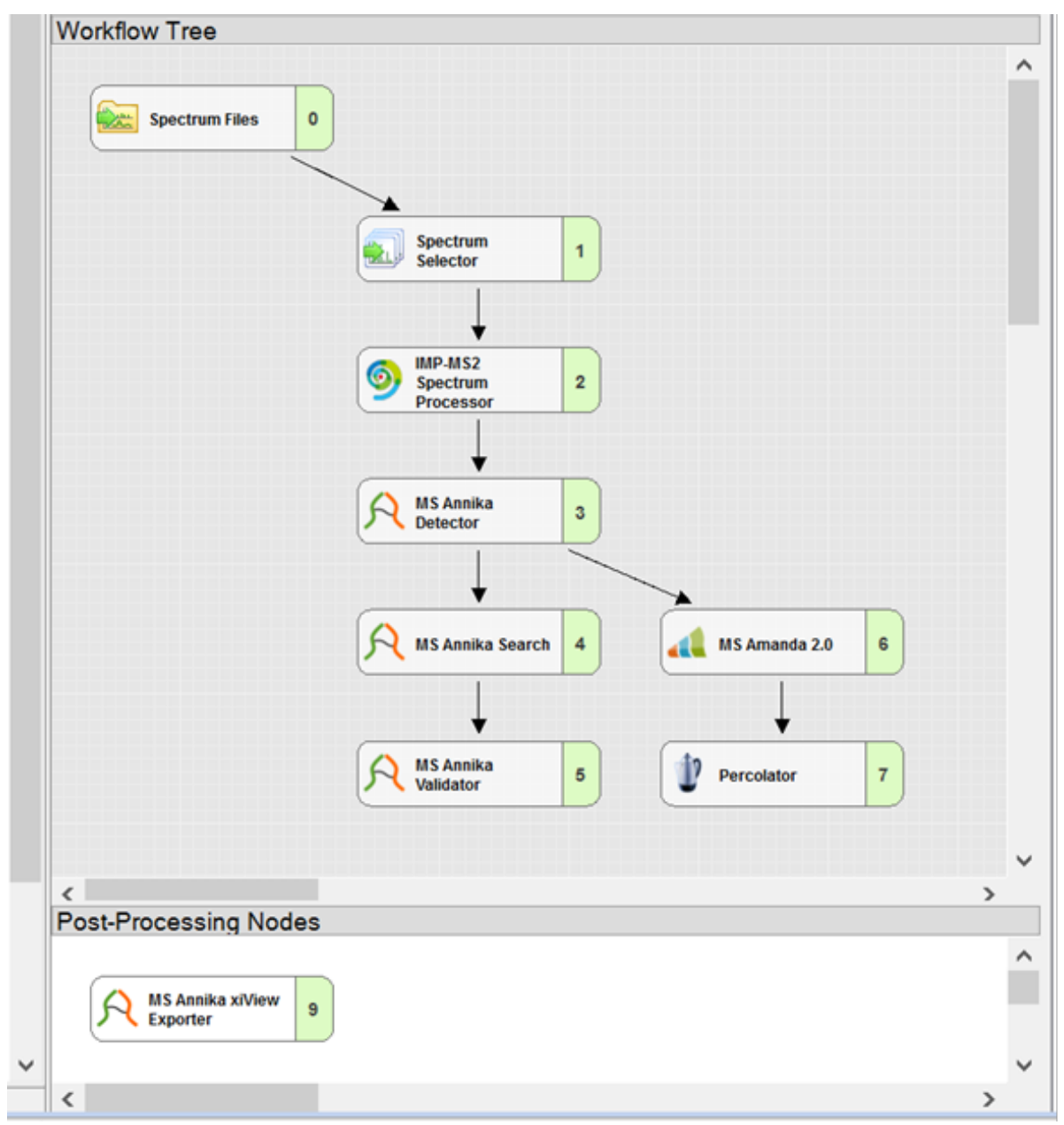

Supplementary Figure 1: The MS Annika Workflow in Proteome Discoverer. Spectra are read from a variety of different formats using the Spectrum Files node. The Spectrum Selector can be used to filter spectra of interest. The optional IMP-MS2 Spectrum processor provides de-isotoping but is not required. The MS Annika Detector identifies potential ion doublets, passes the spectra to the search node if doublets are found, and to a standard peptide identification algorithm if no doublets are found. In this case, we use the MS Amanda database search engine in conjunction with Percolator to identify linear peptides [9], [27]. Spectra with identified ion doublets are searched using the MS Annika Search node. The final combination of crosslink spectrum matches to cross-links as well as multilevel FDR control fall into the scope of the MS Annika Validator node. The optional xiView Exporter node can be used to export cross-links at different confidence levels, which can then be uploaded to xiView for evaluation. 


\section{Evidence Mode}

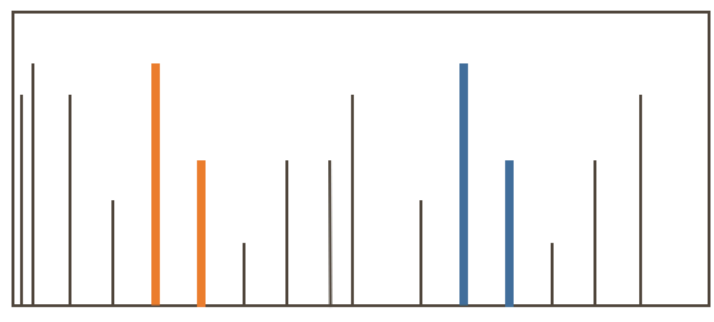

\section{Doublet-Doublet}

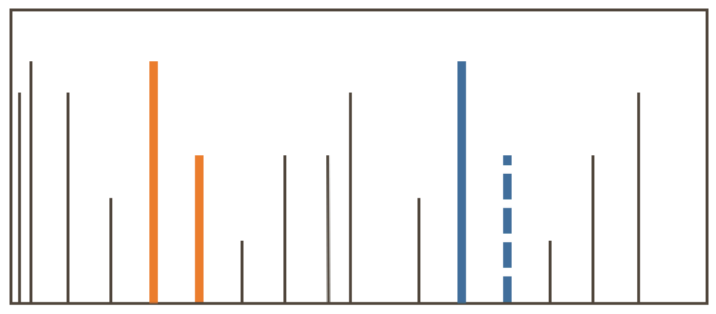

\section{Doublet-Singlet}

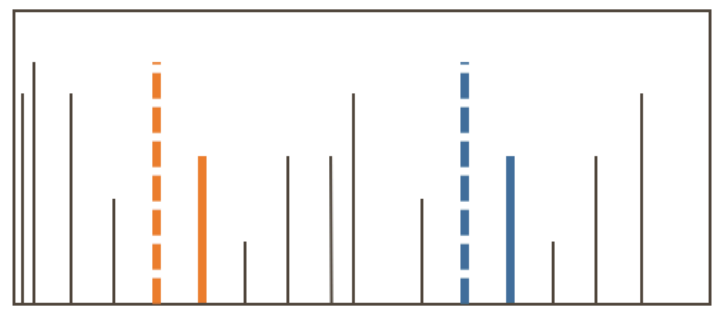

\section{Singlet-Singlet}

Supplementary Figure 2: Evidence Mode of MS Annika. In evidence mode the MS Annika Detector will detect the presence of a cross-link spectrum when a) both doublets are present (doublet-doublet), b) one double is present and the other one is only represented by a single peak (doublet- singlet) or c) the two cross-linked peptides are only represented by a single peak each (singlet-singlet). If ions are missing the MS Annika Detector recalculates them based on the measured precursor mass and on the used cross-linker.

\section{Indication Mode}

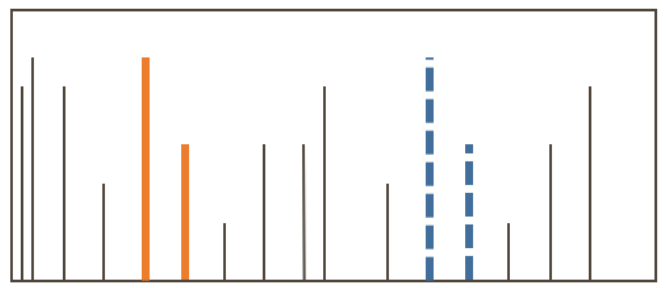

Supplementary Figure 3: Indication Mode of MS Annika. In indication mode the MS Annika Detector looks for doublet peaks with the appropriate distance only. The second doublet will be calculated based on the measured precursor mass and the used cross-linker. 


\begin{tabular}{|l|l|l|l|l|l|l|l|l|l|}
\hline & & & \multicolumn{2}{|l|}{ All Results } & \multicolumn{2}{l}{ 5\% FDR } & \multicolumn{2}{l|}{ FDR } & \\
\hline Linker & $\begin{array}{l}\text { PRIDE } \\
\text { identifier }\end{array}$ & Comment & \# CSMs & $\begin{array}{l}\# \\
\text { Crosslinks }\end{array}$ & $\begin{array}{l}\# \\
\text { CSMs }\end{array}$ & $\begin{array}{l}\# \\
\text { Cross- } \\
\text { links }\end{array}$ & $\begin{array}{l}\# \\
\text { CSMs }\end{array}$ & $\begin{array}{l}\# \\
\text { Cross- } \\
\text { links }\end{array}$ & $\begin{array}{l}\text { Refer- } \\
\text { ence }\end{array}$ \\
\hline DSSO & PXD010796 & $\begin{array}{l}\text { Human DB / } \\
\text { with } \\
\text { Background }\end{array}$ & 28895 & 27059 & 551 & 45 & 382 & 39 & {$[16]$} \\
\hline DSSO & PXD010796 & $\begin{array}{l}\text { Human DB / } \\
\text { no } \\
\text { Background }\end{array}$ & 23667 & 20819 & 931 & 98 & 725 & 67 & {$[16]$} \\
\hline DSAU & PXD018935 & Fraction A & 1566 & 820 & 606 & 159 & 488 & 119 & {$[33]$} \\
\hline DSAU & PXD018935 & Fraction B & 1166 & 672 & 423 & 157 & 344 & 133 & {$[33]$} \\
\hline $\begin{array}{l}\text { BPD- } \\
\text { NHP }\end{array}$ & PXD008975 & & 198197 & 182211 & 9179 & 2651 & 6189 & 1874 & {$[30]$} \\
\hline DSBU & PXD012546 & Fraction R1 & 180294 & 168290 & 3983 & 1902 & 2569 & 1353 & {$[31]$} \\
\hline DSBU & PXD014337 & & 2421 & 1061 & 1613 & 284 & 1397 & 250 & {$[20]$} \\
\hline DSSO & PXD014337 & & 2367 & 906 & 1605 & 228 & 1373 & 209 & {$[20]$} \\
\hline DSSO & PXD011861 & & 13723 & 5908 & 5923 & 785 & 4814 & 632 & {$[17]$} \\
\hline DSSO & PXD022772 & r1 \& r2 & 1464 & 304 & 1188 & 183 & 1018 & 165 & - \\
\hline DSBU & PXD022772 & r1 \& r2 & 1472 & 342 & 1111 & 205 & 943 & 185 & - \\
\hline DSBSO & PXD016963 & & 3146 & 913 & & 224 & & 163 & {$[32]$} \\
\hline
\end{tabular}

Supplementary Table 1: Numbers of identified cross-links and CSMs for different data sets presented in this work. 1\% and 5\% FDR results are based on the FDR estimation in MS Annika. 


\begin{tabular}{|l|l|l|l|l|}
\hline $\begin{array}{l}\text { PXD014337, } \\
\text { DSBU and } \\
\text { DSSO }\end{array}$ & MS Annika & MeroX & XlinkX & pLink \\
\hline MS1 Tolerance & $5 \mathrm{ppm}$ & $5 \mathrm{ppm}$ & $5 \mathrm{ppm}$ & $5 \mathrm{ppm}$ \\
\hline MS2 Tolerance & $10 \mathrm{ppm}$ & $10 \mathrm{ppm}$ & $10 \mathrm{ppm}$ & $10 \mathrm{ppm}$ \\
\hline Enzyme & Trypsin & Trypsin & Trypsin & Trypsin \\
\hline $\begin{array}{l}\text { Missed } \\
\text { Cleavages }\end{array}$ & 3 & 3 & 3 & 3 \\
\hline $\begin{array}{l}\text { Precursor } \\
\text { offset }\end{array}$ & 3 & 3 & 3 & Not available \\
\hline $\begin{array}{l}\text { Cross-link } \\
\text { modification } \\
\text { on }\end{array}$ & $\mathrm{K}$ & $\mathrm{K}$ & $\mathrm{K}$ & K \\
\hline $\begin{array}{l}\text { PTMs } \\
\text { KTM }\end{array}$ & $\begin{array}{l}\text { Carbamidomethyl } \\
\text { (C, Static), } \\
\text { Oxidation (M, } \\
\text { Dynamic) }\end{array}$ & $\begin{array}{l}\text { Carbamidomethyl } \\
\text { (C, Static), } \\
\text { Oxidation (M, } \\
\text { Dynamic) }\end{array}$ & $\begin{array}{l}\text { Carbamidomethyl } \\
\text { (C, Static), } \\
\text { Oxidation (M, } \\
\text { Dynamic) }\end{array}$ & $\begin{array}{l}\text { Carbamidomethyl } \\
\text { (C, Static), } \\
\text { Oxidation (M, } \\
\text { Dynamic) }\end{array}$ \\
\hline Search Mode & Combined Mode & RiseUp & MS2 & MS-Cleavable \\
\hline
\end{tabular}

\begin{tabular}{|l|l|l|l|}
\hline $\begin{array}{l}\text { PXD010796, } \\
\text { with and } \\
\text { without } \\
\text { background }\end{array}$ & MS Annika & MeroX & XlinkX \\
\hline MS1 Tolerance & $10 \mathrm{ppm}$ & $10 \mathrm{ppm}$ & $10 \mathrm{ppm}$ \\
\hline MS2 Tolerance & $20 \mathrm{ppm}$ & $20 \mathrm{ppm}$ & $20 \mathrm{ppm}$ \\
\hline Enzyme & Trypsin & Trypsin & Trypsin \\
\hline $\begin{array}{l}\text { Missed } \\
\text { Cleavages }\end{array}$ & 4 & 3 & 4 \\
\hline $\begin{array}{l}\text { Precursor } \\
\text { offset }\end{array}$ & 3 & 3 & 3 \\
\hline $\begin{array}{l}\text { Cross-link } \\
\text { modification } \\
\text { on }\end{array}$ & K, N-Term & K, N-Term & K, N-Term \\
\hline $\begin{array}{l}\text { PTMs } \\
\text { Carbamidomethyl } \\
\text { (C, Static), } \\
\text { Oxidation (M, } \\
\text { Dynamic) }\end{array}$ & Proteome-wide & $\begin{array}{l}\text { Carbamidomethyl } \\
\text { (C, Static), } \\
\text { Oxidation (M, } \\
\text { Dynamic) }\end{array}$ \\
\hline Search Mode & $\begin{array}{l}\text { Combined Mode } \\
\text { Proteome-wide }\end{array}$ & $\begin{array}{l}\text { MS2_MS2 } \\
\text { mode }\end{array}$ \\
\hline
\end{tabular}

\begin{tabular}{|l|l|}
\hline $\begin{array}{l}\text { PXD018935, } \\
\text { DSAU }\end{array}$ & MS Annika \\
\hline MS1 Tolerance & 4 ppm \\
\hline MS2 Tolerance & $8 \mathrm{ppm}$ \\
\hline Enzyme & Trypsin \\
\hline $\begin{array}{l}\text { Missed } \\
\text { Cleavages }\end{array}$ & 3 \\
\hline
\end{tabular}




\begin{tabular}{|l|l|}
\hline $\begin{array}{l}\text { Precursor } \\
\text { offset }\end{array}$ & 5 \\
\hline $\begin{array}{l}\text { Cross-link } \\
\text { modification } \\
\text { on }\end{array}$ & K, N-Term \\
\hline PTMs & $\begin{array}{l}\text { Carbamidomethyl } \\
\text { (C, Static), } \\
\text { Oxidation (M, } \\
\text { Dynamic) }\end{array}$ \\
\hline Search Mode & Combined Mode \\
\hline
\end{tabular}

\begin{tabular}{|l|l|}
\hline $\begin{array}{l}\text { PXD008975, } \\
\text { BDP-NHP }\end{array}$ & MS Annika \\
\hline MS1 Tolerance & $10 \mathrm{ppm}$ \\
\hline MS2 Tolerance & $20 \mathrm{ppm}$ \\
\hline Enzyme & Trypsin \\
\hline $\begin{array}{l}\text { Missed } \\
\text { Cleavages }\end{array}$ & 4 \\
\hline $\begin{array}{l}\text { Precursor } \\
\text { offset }\end{array}$ & 5 \\
\hline $\begin{array}{l}\text { Cross-link } \\
\text { modification } \\
\text { on }\end{array}$ & K, N-Term \\
\hline PTMs & $\begin{array}{l}\text { Carbamidomethyl } \\
\text { (C, Static), } \\
\text { Oxidation (M, } \\
\text { Dynamic) }\end{array}$ \\
\hline Search Mode & Combined Mode \\
\hline
\end{tabular}

\begin{tabular}{|l|l|}
\hline $\begin{array}{l}\text { PXD012546, } \\
\text { DSBU }\end{array}$ & MS Annika \\
\hline MS1 Tolerance & 4 ppm \\
\hline MS2 Tolerance & $8 \mathrm{ppm}$ \\
\hline Enzyme & Trypsin \\
\hline $\begin{array}{l}\text { Missed } \\
\text { Cleavages }\end{array}$ & 3 \\
\hline $\begin{array}{l}\text { Precursor } \\
\text { offset }\end{array}$ & 3 \\
\hline $\begin{array}{l}\text { Cross-link } \\
\text { modification } \\
\text { on }\end{array}$ & K, N-Term \\
\hline $\begin{array}{l}\text { PTMs } \\
\text { Carbamidomethyl } \\
\text { (C, Static), } \\
\text { Oxidation (M, } \\
\text { Dynamic) }\end{array}$ \\
\hline Search Mode & Combined Mode \\
\hline
\end{tabular}




\begin{tabular}{|l|l|}
\hline $\begin{array}{l}\text { PXD022772, } \\
\text { Peplib } \\
\text { DSSO/DSBU } \\
\text { timsTOF }\end{array}$ & MS Annika \\
\hline MS1 Tolerance & $5 \mathrm{ppm}$ \\
\hline MS2 Tolerance & $10 \mathrm{ppm}$ \\
\hline Enzyme & Trypsin \\
\hline $\begin{array}{l}\text { Missed } \\
\text { Cleavages }\end{array}$ & 3 \\
\hline Precusor offset & 3 \\
\hline $\begin{array}{l}\text { Cross-link } \\
\text { modification } \\
\text { on }\end{array}$ & $\mathrm{K}, \mathrm{N}-$ Term \\
\hline PTMs & $\begin{array}{l}\text { Carbamidomethyl } \\
\text { (C, Static), } \\
\text { Oxidation (M, } \\
\text { Dynamic) }\end{array}$ \\
\hline Search Mode & Combined Mode \\
\hline
\end{tabular}

\begin{tabular}{|c|c|}
\hline $\begin{array}{l}\text { PXD016963, } \\
\text { DSBSO }\end{array}$ & MS Annika \\
\hline MS1 Tolerance & $10 \mathrm{ppm}$ \\
\hline MS2 Tolerance & $20 \mathrm{ppm}$ \\
\hline Enzyme & Trypsin \\
\hline $\begin{array}{l}\text { Missed } \\
\text { Cleavages }\end{array}$ & 4 \\
\hline $\begin{array}{l}\text { Precursor } \\
\text { offset }\end{array}$ & 5 \\
\hline $\begin{array}{l}\text { Cross-link } \\
\text { modification } \\
\text { on }\end{array}$ & $\mathrm{K}, \mathrm{N}$-Term \\
\hline PTMs & $\begin{array}{l}\text { Carbamidomethyl } \\
\text { (C, Static), } \\
\text { Oxidation (M, } \\
\text { Dynamic) }\end{array}$ \\
\hline Search Mode & Combined Mode \\
\hline
\end{tabular}




\begin{tabular}{|l|l|l|}
\hline linker & Estimated CSM FDR & Calculated CSM FDR \\
\hline DSBU (PXD014337, [20]) & $1 \%$ & $1.45 \%$ \\
\hline DSBU (PXD014337, [20]) & $5 \%$ & $4.62 \%$ \\
\hline DSSO (PXD014337, [20]) & $1 \%$ & $2.50 \%$ \\
\hline DSSO (PXD014337, [20]) & $5 \%$ & $6.47 \%$ \\
\hline
\end{tabular}

Supplementary Table 3: CSM level FDRs for MS Annika. Comparison of calculated FDRs based on wrongly identified CSMs from different groups with estimated FDRs for $1 \%$ and $5 \%$ based on the data set of Beveridge and co-workers [20].

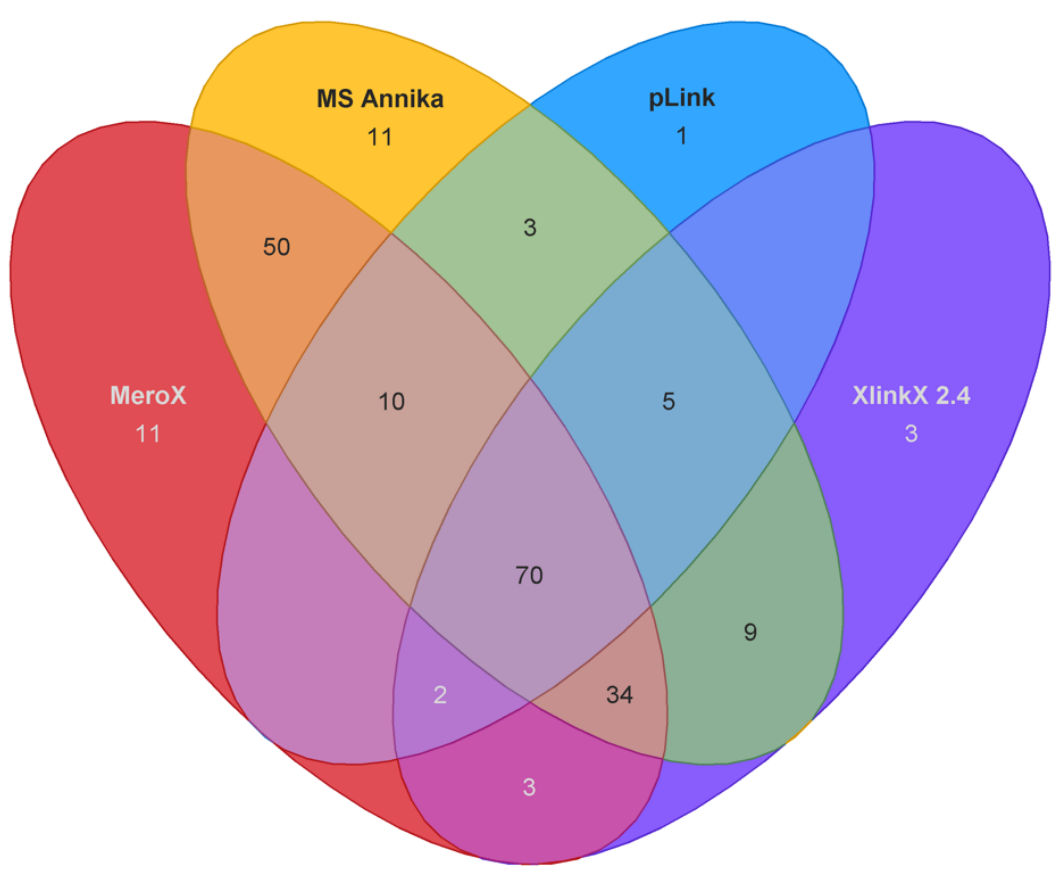

Supplementary Figure 4: Overlap of cross-linkers for DSSO linked peptides. Here the overlap of cross-links within the same group identified at 1\% FDR of four different search engines, namely MS Annika, pLink, XLinkX, and Merox from the data set by Beveridge and co-workers are displayed [20]. 


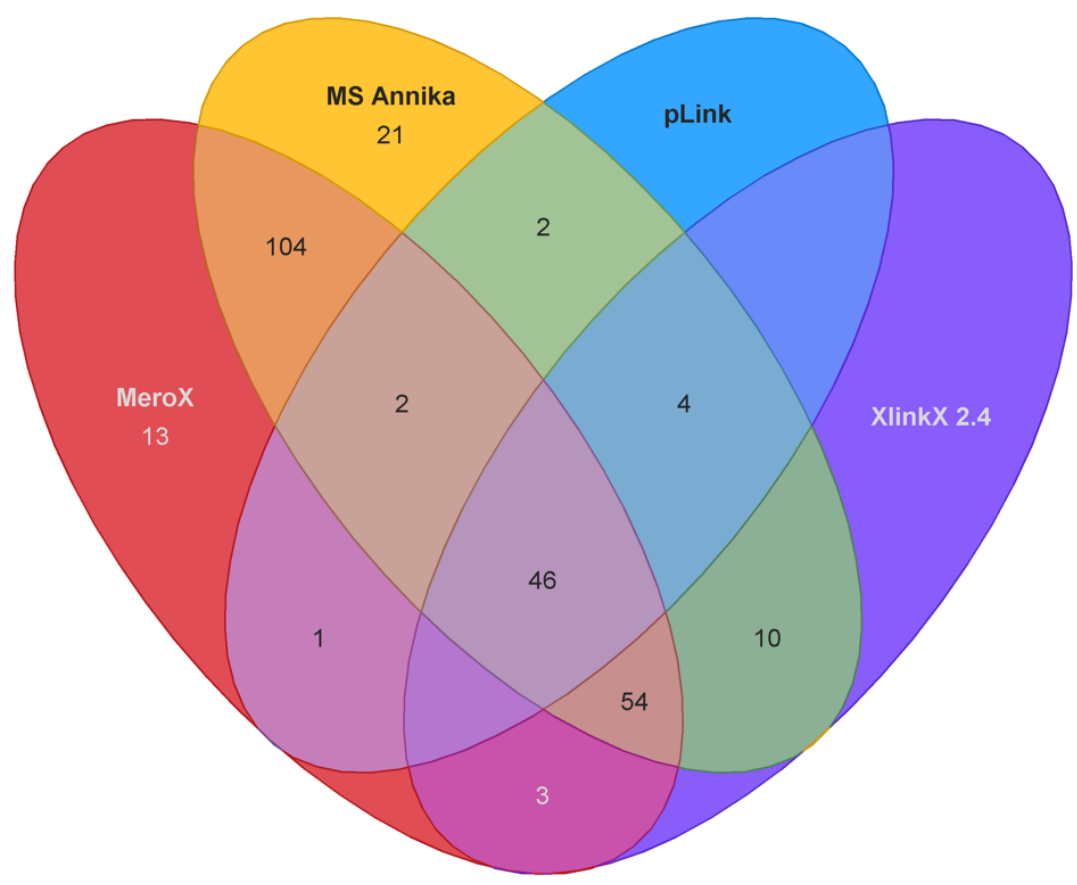

Supplementary Figure 5: Overlap of cross-linkers for DSBU linked peptides. Here the overlap of cross-links within the same group identified at 1\% FDR of four different search engines, namely MS Annika, pLink, XLinkX, and MeroX from the data set by Beveridge and co-workers are displayed [20].
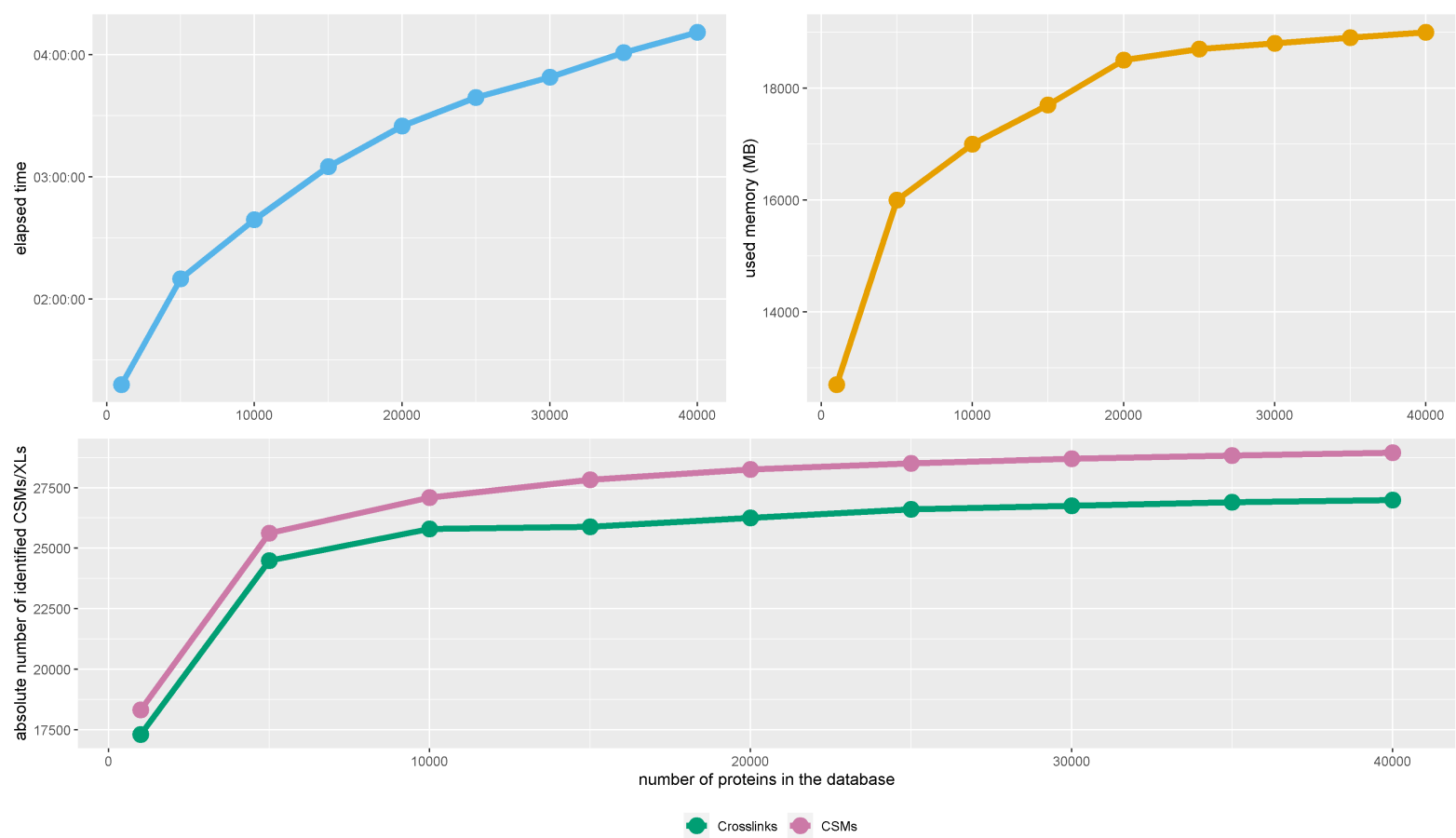

Supplementary Figure 6: Runtime and memory analysis. Randomly selected proteins have been sampled from a human uniprot database including isoforms and common contaminants. Resulting FASTA files ranged from 1000, 5000, to 40000 proteins. Runtimes and approximate memory consumptions are given in blue and orange on the top. On the bottom the total number of identified CSMs and cross-links (without applying any FDR threshold) are depicted to assess the data set size. 


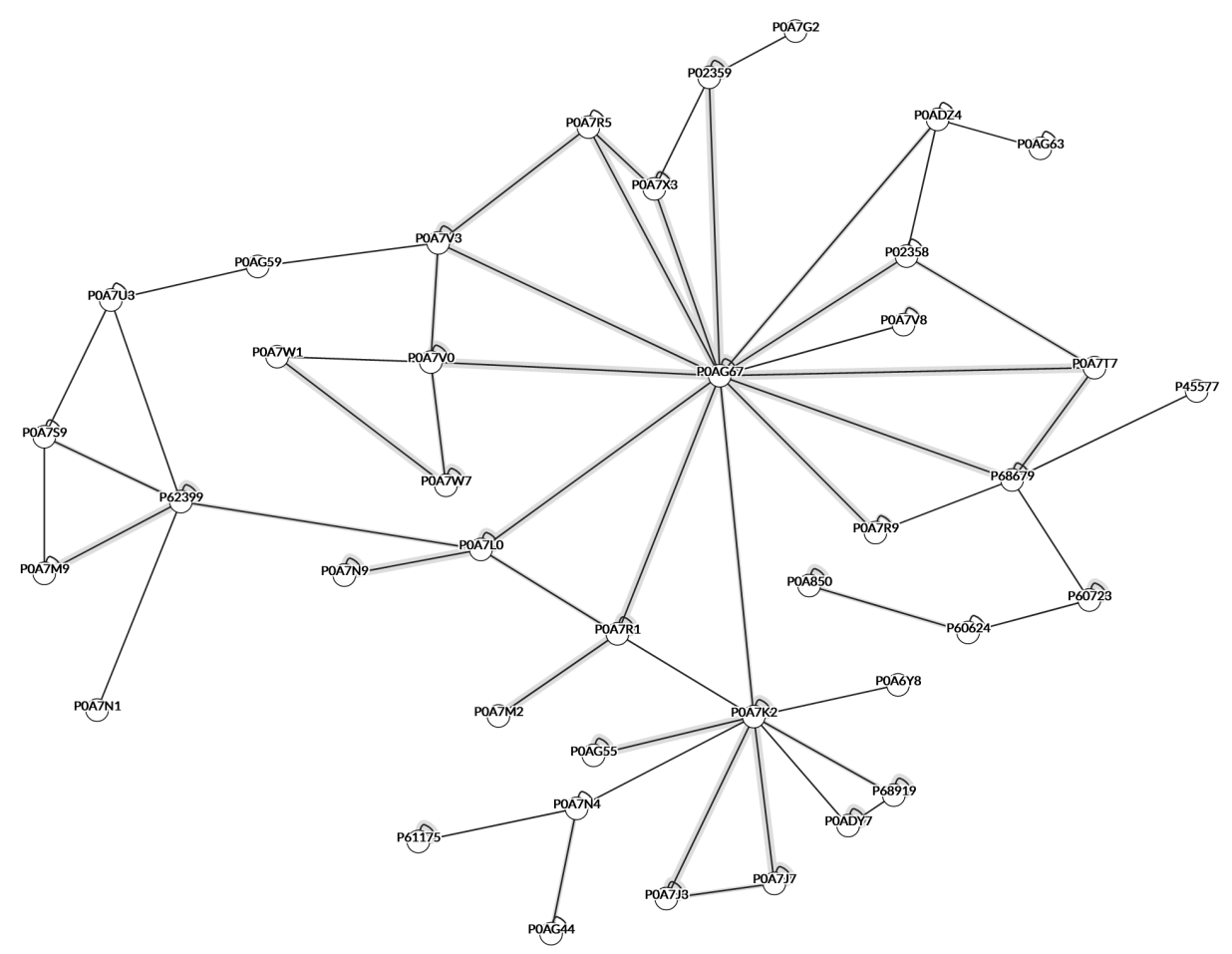

Supplementary Figure 7: Protein interaction network created within xiView, for cross-links that are within the 26 Angstrom distance constraints for DSSO. The protein interaction network was drawn from data published by Stieger and co-workers [2]. 


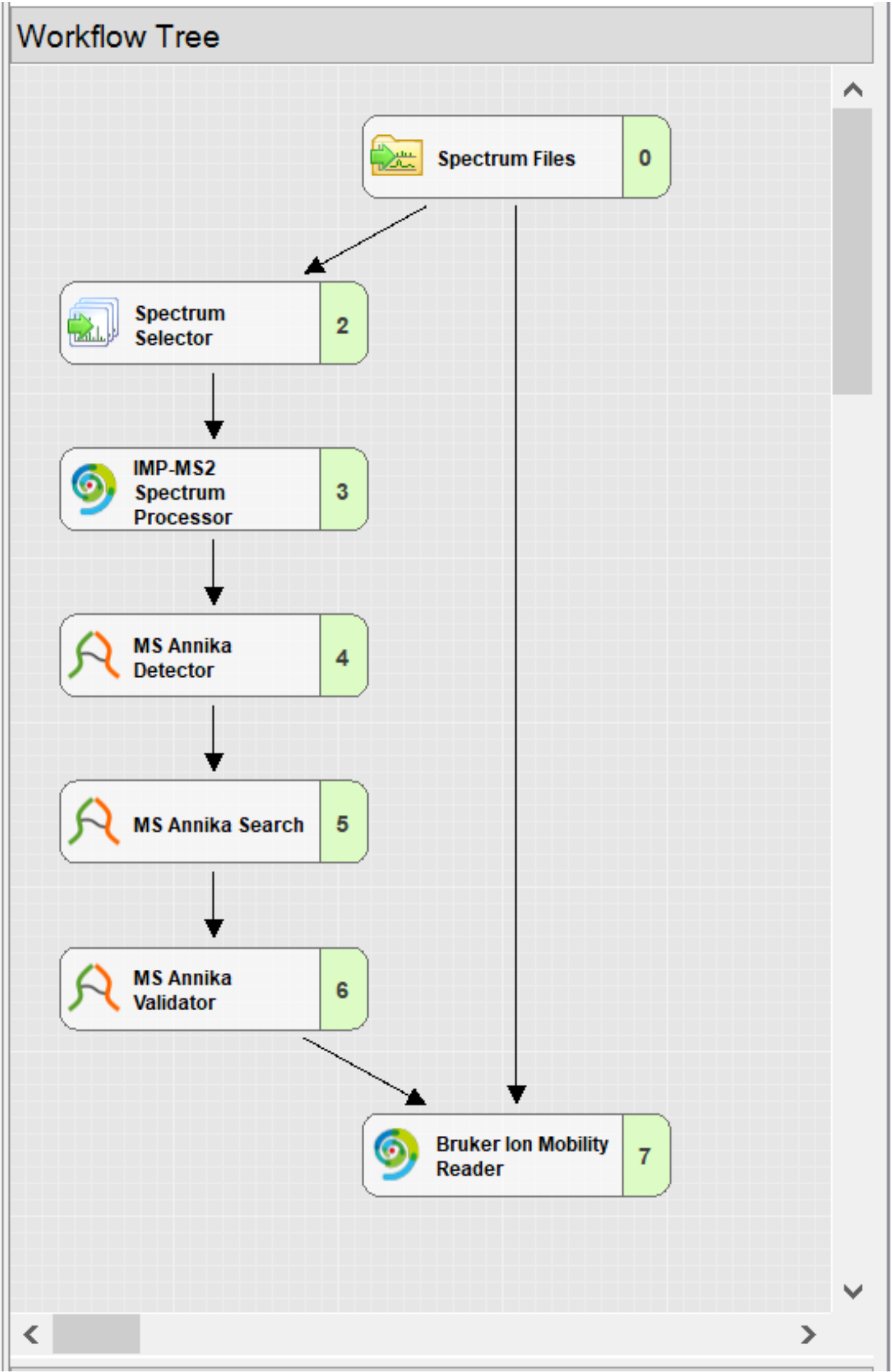

Supplementary Figure 8: Modified Workflow for timsTOF data, using the Bruker lon Mobility Reader. This additional node can be installed during the setup process for MS Annika. 


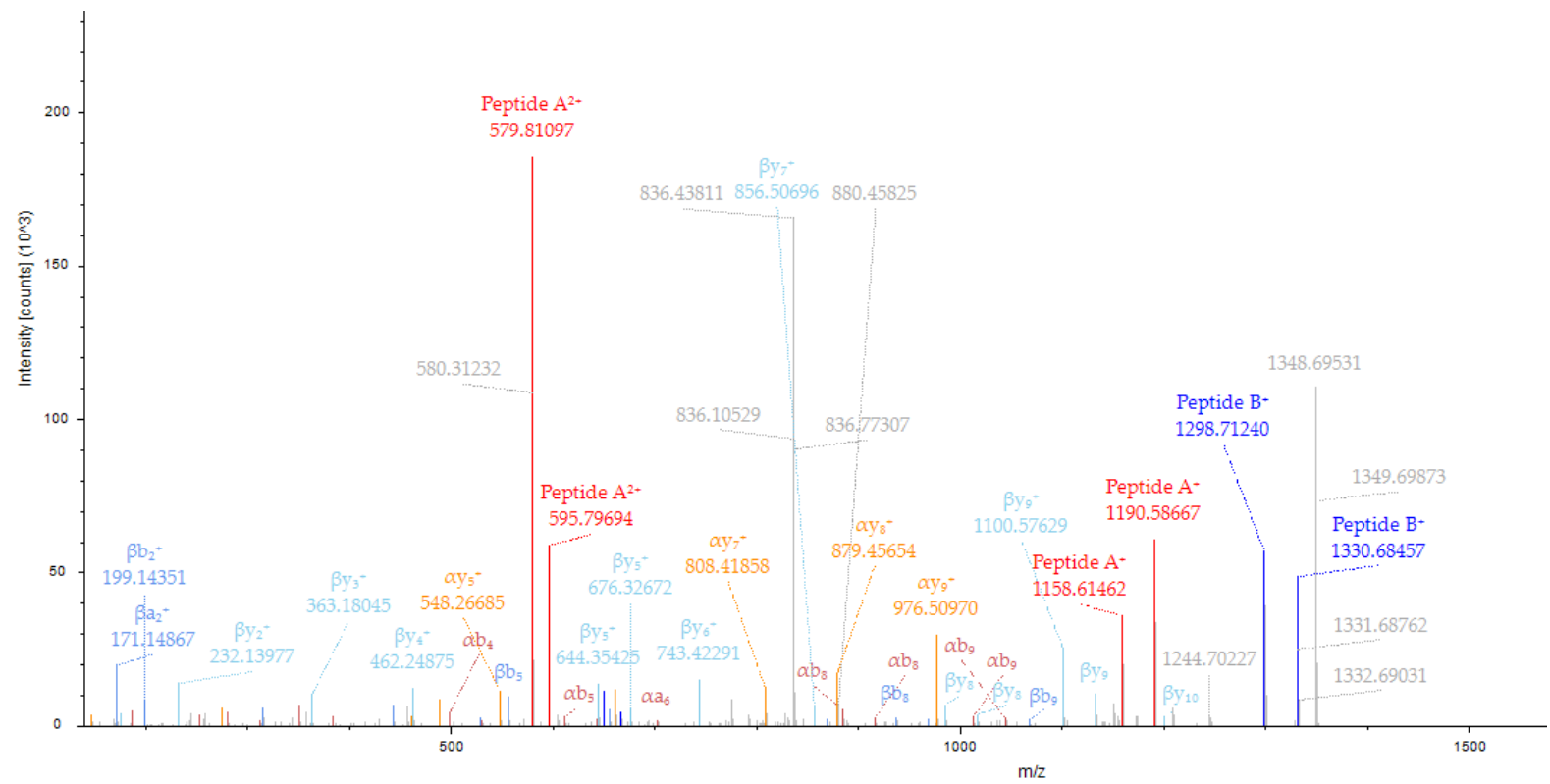

Supplementary Figure 9: Example CSM of the DSSO data set by Beveridge et al. [20], as depicted in Thermo Proteome Discoverer 2.5. This spectrum represents scan number $\# 20799(z=+3, m / z=836.10760)$ with the crosslinked peptides $A$ (KPAFLSGEQK) and B (VVDELVKVMGR). Cross-linking positions are marked in bold. Peptides have been identified using Combined Mode, having a score of 660.3.

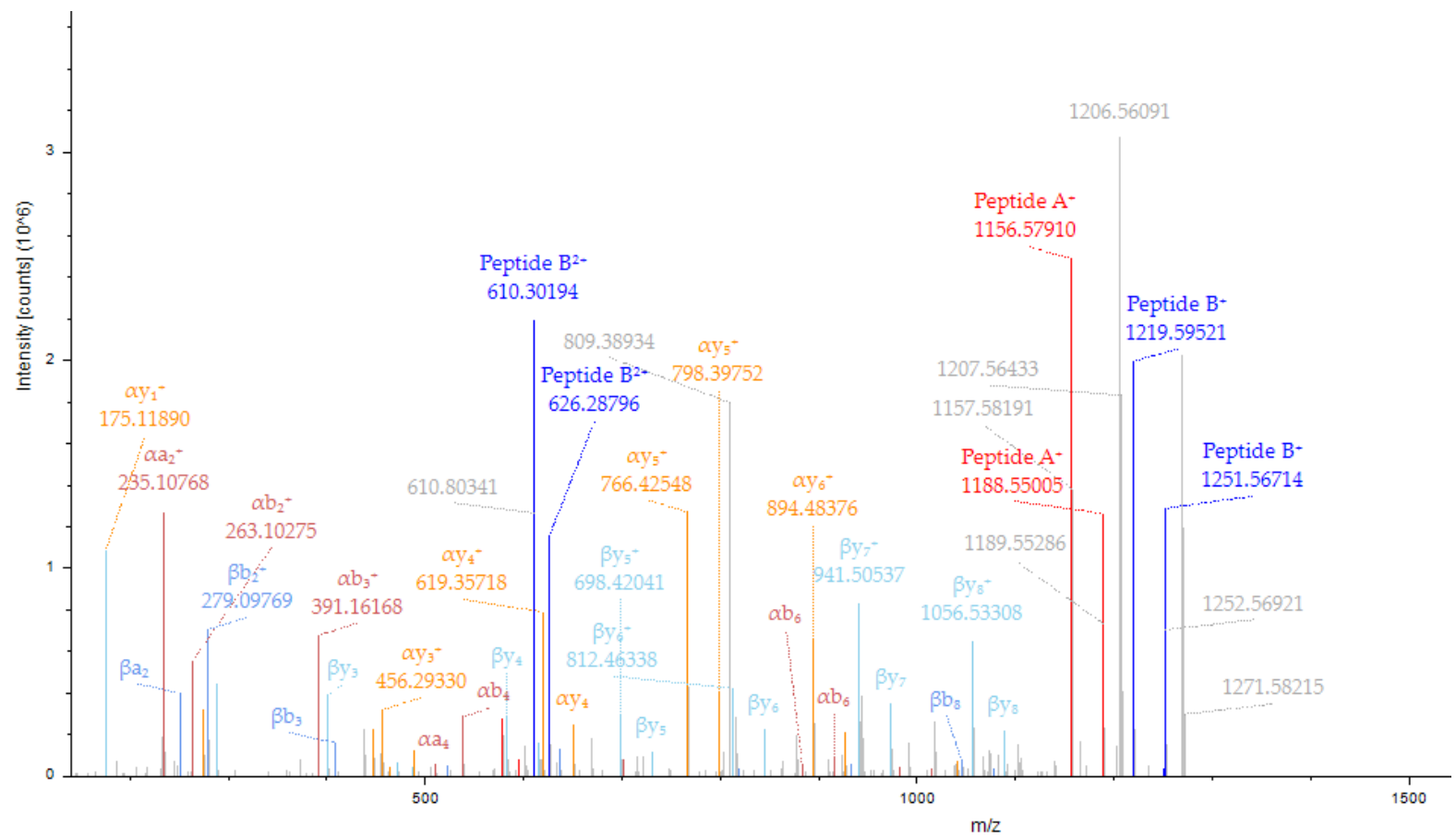

Supplementary Figure 10: Example CSM of the DSSO data set by Beveridge et al. [20], as depicted in Thermo Proteome Discoverer 2.5. This spectrum represents scan number \#20983 ( $z=+3, m / z=809.0556)$ with the crosslinked peptides $A$ (DFQFYKVR) and B (YDENDKLIR). Cross-linking positions are marked in bold. Peptides have been identified using Combined Mode, having a score of 465.9. 